\title{
Meerschweinchen stressarm behandeln und Unwohlsein erkennen
}

Barbara M. Schneider

Meerschweinchen sind sehr stressanfällig. Stress äußert sich bei diesen Tieren jedoch, ähnlich wie Schmerzen, oft nur subtil. Daher muss der behandelnde Tierarzt die Tiere aufmerksam beobachten. Zudem kann er mit wenigen, simplen Maßnahmen dafür sorgen, den Stress der Behandlung zu reduzieren.

\section{Normalverhalten und Vermeidung von Stress}

Ein solides Wissen um das Normalverhalten und die Ansprüche von Meerschweinchen sind Grundvoraussetzung für das Erkennen und Vermeiden von Stress. Anhand des Verhaltens können auch bei einem Meerschweinchen die besten Rückschlüsse auf sein Befinden gezogen werden. Im Folgenden werden die für einen praktischen Tierarzt relevantesten Verhaltensweisen mit ihrer Bedeutung für den Umgang mit den Meerschweinchen aufgeführt.
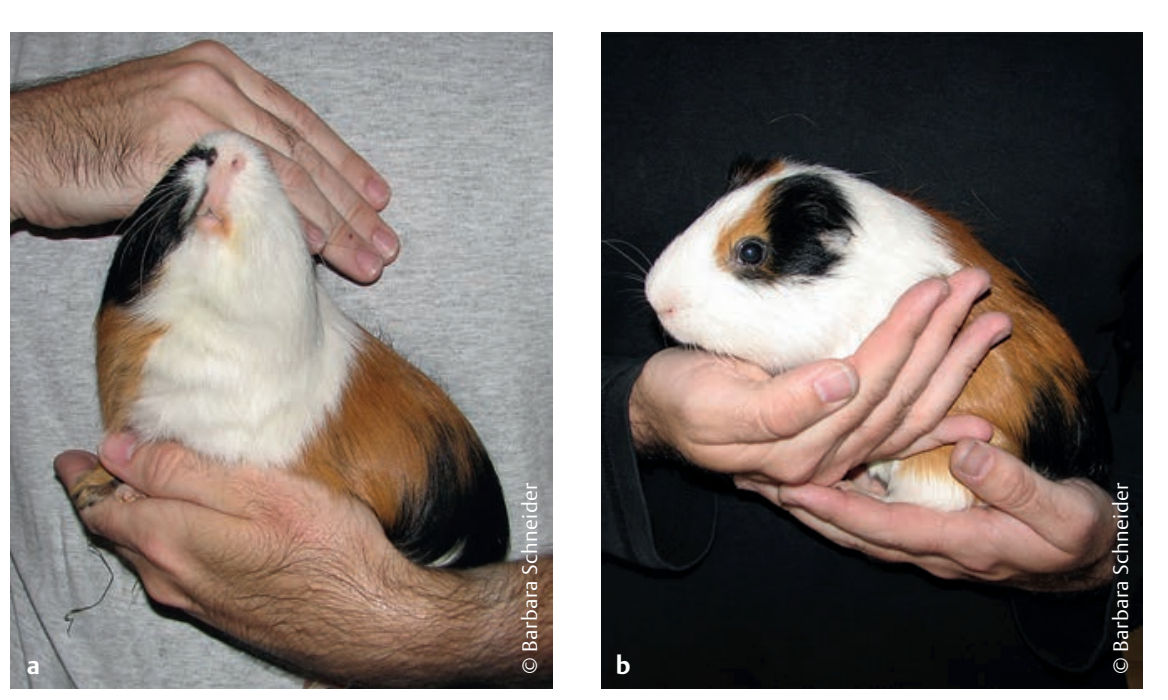

Abb. 1 a Deutliches Drohverhalten beim Streicheln über Kopf und Rücken. b Das Meerschweinchen aus Abb. 1a sitzt relativ entspannt in der Hand, wenn es nicht gestreichelt wird.

\section{Bindungspartner}

Meerschweinchen sind obligat sozial. Das bedeutet, dass sie zwingend mit anderen Meerschweinchen zusammenleben müssen, um sich wohlzufühlen. Die soziale Organisation von Meerschweinchengruppen ist dabei unter anderem von der Gruppengröße und der Populationsdichte abhängig [5]. Zwischen befreundeten Tieren kann es zu einer sehr engen Bindung kommen. Dieses sogenannte Bindungsmeerschweinchen ist für seinen Partner in stressigen Situationen eine wichtige emotionale Stütze. Es hilft ihm, diese zu meistern $[3,6]$. Dieses Phänomen bezeichnet man auch als „social support“.

Für die tierärztliche Praxis bedeutet dies, dass dem Besitzer empfohlen werden muss, den Bindungspartner des Patienten mitzubringen. Dies ist besonders dann anzuraten, wenn der Patient längere Zeit in der Klinik verweilen muss. Auch, wenn bei Weibchen sogar die Anwesenheit eines beliebigen bekannten Tieres hilfreich ist [3], sollte dennoch immer idealerweise der beste Freund des betroffenen Meerschweinchens dabei sein.

\section{Fellpflege}

Meerschweinchen zeigen, im Gegensatz zu den meisten anderen sozial lebenden Spezies, kein soziales Putzen (Allogrooming). Kommt es dennoch vor, dass Meerschweinchen ihre Artgenossen beknabbern oder belecken, ist dies in der überwiegenden Zahl der Fälle pathologisch und sollte näher untersucht werden. Da Meerschweinchen keine soziale Fellpflege betreiben, empfinden sie auch Streicheln nicht als angenehm. Häufig wird das Streicheln über Kopf und $\mathrm{Na}-$ cken daher auch mit einem Schnauzeheben $(\triangleright$ Abb. 1$)$ oder gar einem Wegdrücken der Hand beantwortet.

Meerschweinchen sollten daher vom Tierarzt bzw. seinen Mitarbeitern nicht gestreichelt werden. Auch wenn kein deutliches Drohverhalten gezeigt wird, ist davon auszugehen, dass diese Art von Körperkontakt nicht als positiv empfunden wird.

\section{Droh- und Abwehrverhalten}

Wenn Meerschweinchen gestresst sind, können sie ein ganzes Repertoire an Droh- und Abwehrverhalten zeigen. Beim Schnauzeheben handelt es sich beispielsweise um explizites Drohverhalten. Dieses wird von Besitzern häufig als positive Interaktion (Aufforderung zum Streicheln) fehlinterpretiert, vor allem, wenn es in direktem Zusammenhang mit dem Streicheln gezeigt wird.

Neben dem Schnauzeheben zeigen Meerschweinchen auch folgendes Drohverhalten:

- Wetzen der Schneidezähne

- gesträubtes Fell

- Aufreißen des Maules

- Zähneklappern 
- gegen den Gegner gerichtetes Gähnen

- Kreischen

- steifes Treten mit den Hinterbeinen

- Hervordrücken der Hoden (Blitzen) bei männlichen Tieren

- Ausschlagen, eventuell mit gleichzeitigem Kreischen, Quietschen oder Abwehrharnen

Vor allem das Zähneklappern ist dabei eine deutliche Angriffswarnung. Abwehrharnen wird, ebenso wie Beißen, von weiblichen Tieren in der Regel häufiger gezeigt als von männlichen.

\section{konkret}

Werden während der Behandlung

Droh- und Abwehrverhaltensweisen gegenüber dem Praxispersonal gezeigt, sollte umgehend versucht werden, den Stress für den Patienten zu reduzieren.

\section{Geruchliche Kommunikation}

Wie alle kleinen Nagetierspezies kommunizieren Meerschweinchen sehr stark über Gerüche. Sowohl die Gruppenmitglieder als auch das Revier werden am Geruch erkannt. Aus diesen Gründen sind Meerschweinchen in einer geruchlich fremden Umgebung schnell gestresst. Die Transportkiste sollte daher immer mit benutzter Einstreu aus dem
Heimatkäfig des Patienten befüllt sein sowie ein bekanntes und beliebtes Häuschen beinhalten. Zudem sollten vor dem Handling die Hände von Tierarzt und Helfer kurz mit vom Patienten benutzter Einstreu eingerieben werden.

\section{Körpersprache}

Auch anhand der Körpersprache lässt sich feststellen, ob ein Meerschweinchen gestresst ist. Die Alarmhaltung von Meerschweinchen ist ein Strecken des Körpers mit nach vorne gerichtetem Kopf. Das Tier macht sich dabei bereit zur Flucht. Es kann zu plötzlichen, explosiven Bewegungen des Meerschweinchens kommen. Bei Furcht vor einem Beutegreifer kann sich ein Meerschweinchen auch größer machen mit durchgestreckten Vorderbeinen und angehobenem Körper. Meerschweinchen sind allerdings im Allgemeinen gegenüber Fressfeinden eher wenig wehrhaft. Daher zeigen sie häufig „Freezing“, also ein sogenanntes Totstellen, um potenzielle Beutegreifer abzuwehren. Die Tiere können dabei sogar auf den Rücken rollen und reglos liegen bleiben. Meerschweinchen fühlen sich nur dann wirklich sicher, wenn sie jederzeit in einen Unterschlupf flüchten können. Auf freien Flächen bewegen sie sich nur ungern. Sie zeigen eine ausgeprägte Thigmotaxis. Dies bedeutet, dass sie sich vor allem in direkter Nähe von Strukturen wie Häuschen,

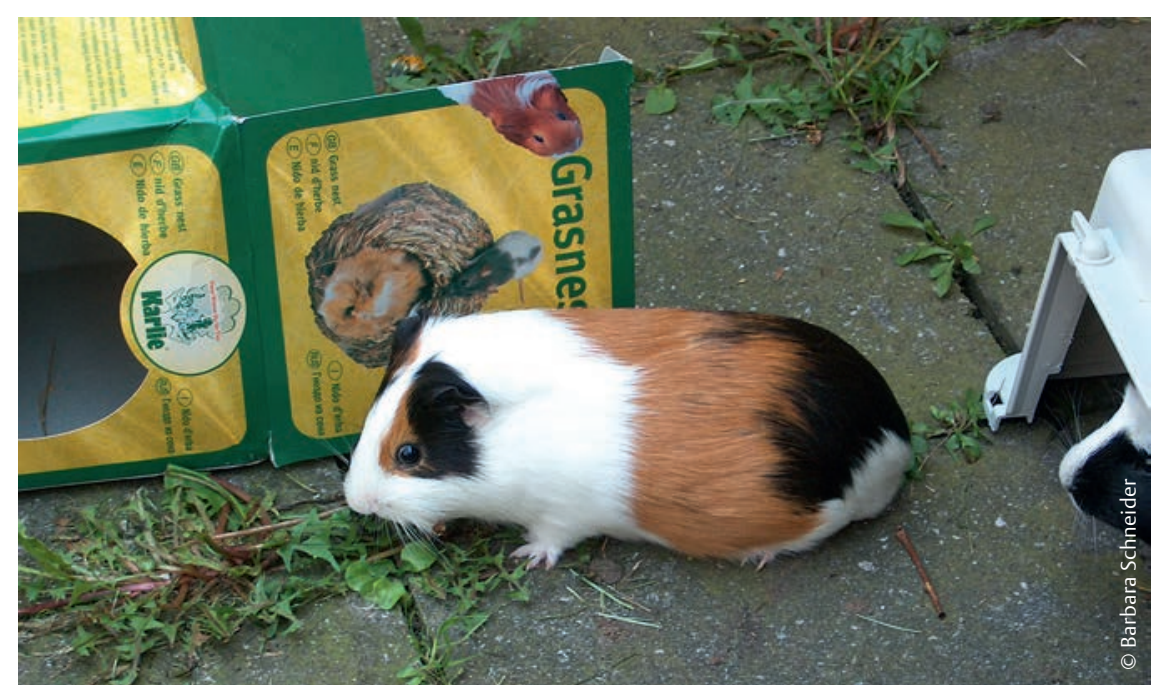

Abb. 2 Thigmotaxis bei sichtlich gestresstem Meerschweinchen.
Wänden oder anderen Gegenständen aufhalten ( $\triangleright$ Abb. 2).

Geht ein Meerschweinchen in die Alarmhaltung, d.h. ein Strecken des Körpers mit nach vorne gerichtetem Kopf, muss es davor bewahrt werden, sich durch plötzliche, hektische Sprünge zu verletzen.

Wird dagegen das Freezing in der Praxis beobachtet, so sollte dem Meerschweinchen nach Möglichkeit die Gelegenheit gegeben werden, sich in einem sicheren Unterschlupf zurückzuziehen und zu erholen. Dies ist vor allem wichtig, wenn noch weitere Stressanzeichen zu erkennen sind ( $\bullet$ Abb. 3 ).

Bei stationärer Aufnahme, idealerweise auch bei der Untersuchung auf dem Behandlungstisch, muss ein geeigneter Unterschlupf zwingend als Rückzugsort zur Verfügung gestellt werden. Sobald notwendige Untersuchungen beendet sind, müssen Meerschweinchen sofort die Gelegenheit haben, sich an einen sicheren Ort zurückzuziehen.

\section{Weitere speziesspezifische Besonderheiten}

\section{Lärmempfindlichkeit}

Das Hörvermögen von Meerschweinchen wurde in vielen verschiedenen Studien mit den unterschiedlichsten Methoden dokumentiert. Die beste Sensitivität liegt

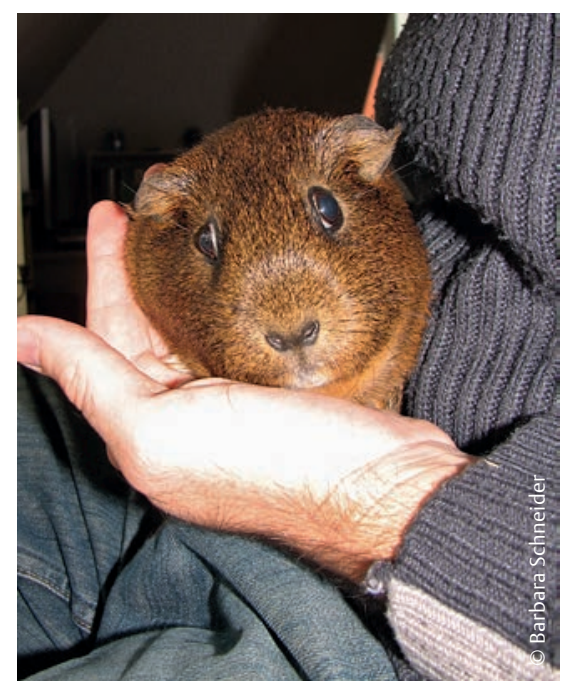

Abb. 3 Meerschweinchen in Vorstufe zum Freezing. Beachte die durch den erhöhten Blutdruck hervorgetretenen Augen sowie die stark erweiterten Pupillen. 
dabei im Bereich 4-8 kHz. Sie können aber auch Ultraschalllaute von bis zu $46 \mathrm{kHz}$ hören [2]. Insgesamt ist für den Tierarzt von Bedeutung, dass die Meerschweinchen sensibler auf akustische Stimuli reagieren als andere kleine Heimtiere. Zudem ist die Latenzzeit, bis die Tiere nach einem entsprechenden Geräusch wieder Normalverhalten zeigen, länger als bei den meisten anderen Spezies.

Auf diese Lärmempfindlichkeit muss auch in der Tierarztpraxis und Klinik Rücksicht genommen werden. Insgesamt muss der Lärmpegel im Wartezimmer und Behandlungsraum für Meerschweinchen niedrig gehalten werden. Dies bedeutet eventuell, dass Meerschweinchen mit ihren Besitzern in einem getrennten, ruhigen Wartezimmer warten.

\section{.konkret}

Bei stationärem Aufenthalt muss darauf geachtet werden, die Meerschweinchen im ruhigsten Teil der Klinik unterzubringen.

\section{Schreckhaftigkeit}

Als kleine Beutetiere sind Meerschweinchen vor allem von plötzlichen Bewegungen von oben gestresst, da sie instinktiv Beutegreifer aus der Luft fürchten. Aus diesem Grund ist es essenziell, dass Meerschweinchen niemals direkt auf dem Boden abgestellt werden. Dies gilt zum einen für den Behandlungsraum, in dem der Transportkäfig immer erhöht stehen sollte. Aber auch im Wartezimmer müssen Meerschweinchenhalter dazu angehalten werden, ihre Tiere erhöht abzustellen, beispielsweise auf ihrem Schoß, auf einem Stuhl etc. Einfache Schilder im Wartezimmer können die Besitzer darauf hinweisen. Ebenso helfen Handtücher, die als Bedeckung für die Transportbox die Sicht einschränken, den Meerschweinchen mehr Sicherheit zu vermitteln.

\section{Anzeichen für Schmerzen}

Für kleine Beutetiere wie Meerschweinchen ist es essenziell, dass sie Schmerzen und Leiden nicht oder nur sehr subtil zeigen. Schließlich sind schwach oder

\section{Maßnahmen zur Stressreduktion in der Tierarztpraxis}

- Der Transportkäfig sollte im Wartezimmer erhöht abgestellt und mit einem Handtuch bedeckt werden.

- Besitzer sollten gebeten werden, den Bindungspartner des zu behandelnden Meerschweinchens mitzubringen. Dies ist besonders bei einem längeren Aufenthalt wichtig ( $\triangleright$ Abb.4).

- Auf dem Behandlungstisch sollte ein warmes Handtuch liegen, um die Rutschsicherheit zu erhöhen und den Wärmeverlust zu vermindern, da die Idealtemperatur für Meerschweinchen bei $20^{\circ} \mathrm{C}$ liegt [4].

- Vor dem Handling sollten die Hände von Tierarzt und Helfer kurz mit vom Patienten benutzter Einstreu eingerieben werden.

- Meerschweinchen sollten nicht vom Tierarzt oder seinen Mitarbeitern gestreichelt werden.

- Nach der Untersuchung der Maulhöhle sollte, wenn möglich, Heu und etwas Frischfutter angeboten werden.

- Bei Droh- und Abwehrverhalten sollte die Untersuchung vorrübergehend unterbrochen und dem Meerschweinchen eine Rückzugsmöglichkeit zur Erholung geboten werden.

- Die Stimmlage des Praxispersonals sollte leise und ruhig sein. Bewegungen müssen sanft bleiben und dürfen niemals plötzlich von oben kommen.

- Ein sicheres Handling der Tiere mit Umfassen des Schultergürtels mit der einen und Stützung des Beckens mit der anderen Hand vermittelt Sicherheit. Bei oraler Medikamentengabe oder Untersuchung der Mundhöhle kann das Meerschweinchen einhändig am Schultergürtel fixiert und angehoben werden. Die Hinterbeine müssen dabei auf einer festen, rutschsicheren Unterlage ruhen.

- Bei stationärer Aufnahme muss ein geeigneter Unterschlupf zwingend zur Verfügung gestellt werden. Meerschweinchen sind im ruhigsten Teil der Klinik und auf Augenhöhe mit dem Behandler unterzubringen.

krank wirkende Tiere die leichteste Beute von Fressfeinden.

Eine genaue Beobachtung des Verhaltens ist zur Schmerzerkennung nötig.

Bei Eingriffen, bei denen zu vermuten ist, dass sie schmerzhaft sind, muss bei Meerschweinchen auch ohne erkennbare Schmerzzeichen immer eine rechtzeitige, ausreichende Analgesie erfolgen. Eine gute Analgesie erhöht nicht nur das Wohlbefinden des Tieres, sondern hilft auch bei der Erholung nach einer Narkose. Meerschweinchen, die eine gute analgetische Versorgung erhalten haben, kehren schneller zum Normalverhalten zurück und fressen auch schneller nach einer Operation. Letzteres hilft, eine lebensbedrohliche gastrointestinale Blockade zu verhindern [1].

Nach Bradley Bays sind im Folgenden einige Anzeichen für Schmerzen bei Meerschweinchen aufgezählt [1]:
- Anorexie

- Absatz von wenigen, kleineren oder keinen Kotboli

- Polydipsie, v.a. bei gastrointestinalem Schmerz

- erniedrigte Körpertemperatur

- sich kühl anfühlende Ohren und Gliedmaßen

- Quietschen bei Palpation einer schmerzhaften Stelle

- Schonhaltung nach Palpation einer schmerzhaften Stelle

- schnelles, flaches Atmen

- Kauen an betroffener Stelle, evtl. mit Alopezie einhergehend

- angestrengter Gesichtsausdruck mit hervortretenden Augen

- Drücken des Abdomens auf den Boden

- Kopf vorgestreckt halten

- leerer, unfokussierter Blick und/oder halbgeschlossene Augen

- Immobilität

- blasse Schleimhäute

- Aggression bei normalerweise freundlichem Tier 


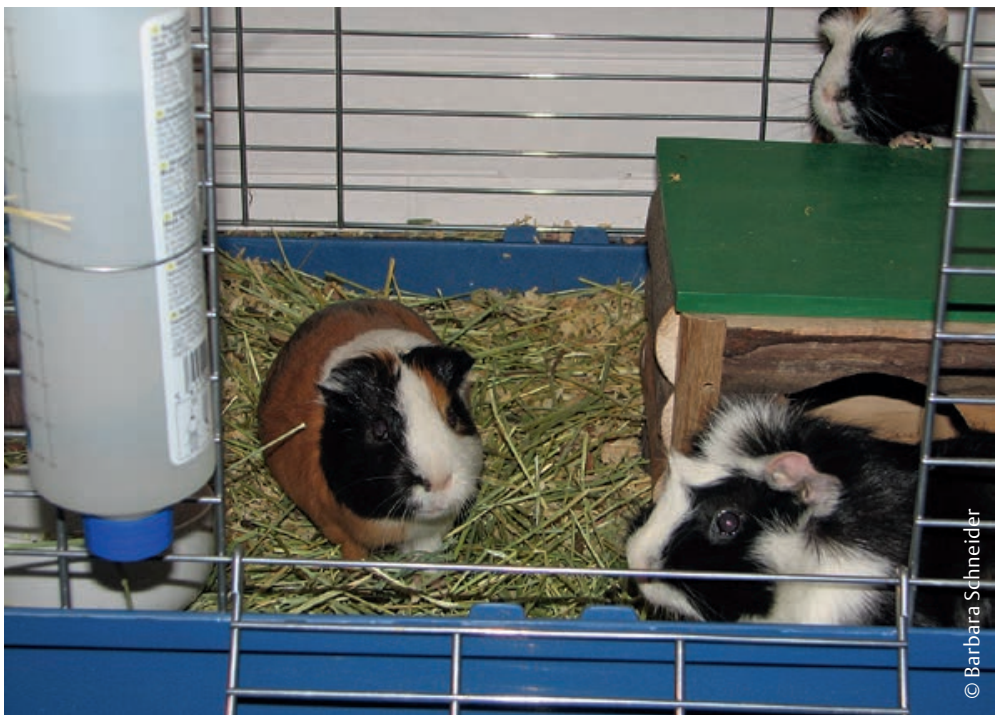

Abb. 4 Komplette Dreiergruppe an Meerschweinchen im Heimatkäfig in der Klinik. Der eigentliche Patient ist das Meerschweinchen hinter dem Häuschen, sein Bindungspartner ist vorne links zu sehen.

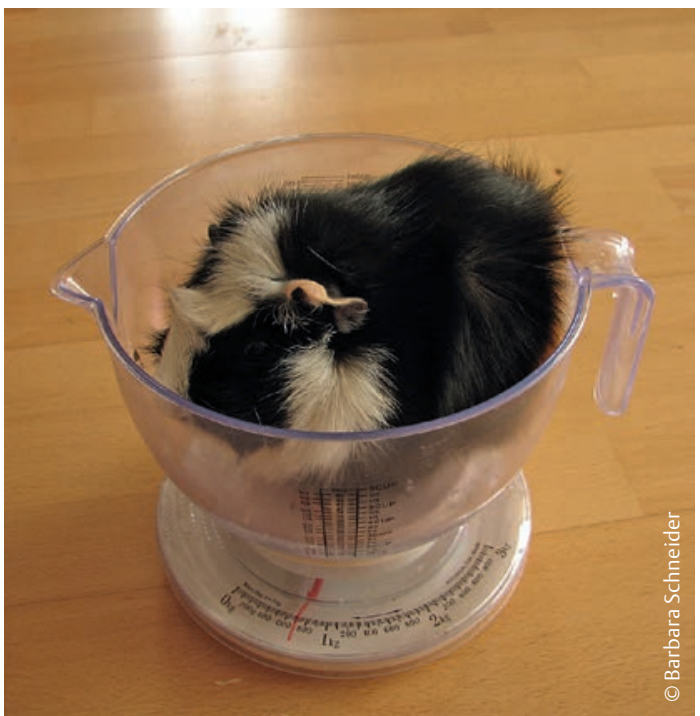

Abb. 5 Meerschweinchen mit bekannten Zahnproblemen beim wöchentlichen Wiegen durch den Besitzer.
- Lethargie, mangelnde Abwehr beim
Handling

\section{Anzeichen für Zahnprobleme}

Zahnfehlstellungen und Zahnprobleme sind bei den Meerschweinchen eines der häufigsten Probleme, weshalb die Tiere in der Praxis vorstellig werden. Auch hier sind die Anzeichen vor allem anfangs oft nicht sehr deutlich. Daher müssen Besitzer zwingend darin geschult werden, Zahnprobleme frühzeitig zu erkennen. Immer, wenn Zahnprobleme vermutet werden oder schon einmal aufgetreten sind, sollten die Besitzer u.a. dazu angehalten werden, ihre Tiere regelmäßig zu wiegen, da ein Gewichtsverlust das erste Zeichen für eine Verschlechterung sein kann ( Abb.5).

Häufige Anzeichen für Zahnprobleme sind nach Bradley Bays u.a. [1]:

- Anorexie oder Dsyphagie

- Gewichtsverlust

- Hypersalivation

- periorale Dermatitis oder Dermatitis in der Kinn- und Halsregion

- mangelndes Putzen sowie Flecken auf den Vordergliedmaßen

- „Bepföteln“ des Maules

- Zähneknirschen
- verlangsamtes Fressen

- vermehrtes Kauen auf einer Seite

- Polydipsie

- Aufnehmen von Futter und Fallenlassen, ohne es zu fressen

- Vermeidung bevorzugter Nahrung

\section{Fazit}

Meerschweinchen sind sehr sensible Patienten, bei denen Stress und Schmerzen nicht immer deutlich zu erkennen sind. Um den Stress für sie zu reduzieren, muss der behandelnde Tierarzt nicht nur seinen Umgang mit den Tieren entsprechend tierfreundlich gestalten, sondern auch die Besitzer über die Ansprüche der Meerschweinchen informieren und sie im Erkennen von Stressanzeichen schulen.

Online zu finden unter

https://doi.org/10.1055/s-0043-113689

\section{Literatur}

1 Bradley Bays T. Guinea pig behavior. In: Bradley Bays T, Lightfoot T, Mayer J, eds. Exotic Pet Behavior: Birds, Reptiles, and Small Mammals. St. Louis: Elsevier; 2006
2 Heffner R, Heffner H, Masterson B. Behavioral measurements of absolute and frequency difference thresholds in guinea pig. J Acoust Soc Am 1971; 49: 1888-1895

3 Kaiser S, Kirtzeck M, Hornschuh G et al. Sex-specific difference in social support - a study in female guinea pigs. Physiol Behav 2003; 79 (2): 297-303

4 Lee Y. Guinea pigs. In: Tynes VV, ed. Behavior of Exotic Pets. Sussex, UK: Wiley Blackwell; 2010

5 Sachser N. Different forms of social organization at high and low population densities in guinea pigs. Behaviour 1986; 97 : 253-272

6 Sachser N, Dürschlag M, Hirzel D. Social relationships and the management of stress. Psychoneuroendocrinology 1998; 23: 891-904

\section{Dr. Barbara M. Schneider}

Fachtierärztin für Verhaltenskunde

54 Ridge Street

Gordon, NSW 2072

Australien

b.schneider@verhalten-schneider.de

www.verhalten-schneider.de 